IP Periodica Polytechnica Electrical Engineering and Computer Science

60(1), pp. 60-64, 2016

DOI: 10.3311/PPee.9042

Creative Commons Attribution (i)

RESEARCH ARTICLE

\section{Indoor Positioning Using Linearly and Circularly Polarized Antennas}

\author{
Zoltán Attila Szalay ${ }^{1}$, Lajos Nagy ${ }^{1 *}$
}

Received 21 September 2015; accepted 02 January 2016

\begin{abstract}
The article deals with the possibility of combination of linear and circular polarized antennas in case of indoor positioning techniques based on signal strength measurement. We have investigated the distribution of the received power by a quasithree-dimensional, own developed ray tracing model and by measurement in LoS (Line of Sight) and NLoS (Non Line of Sight) scenarios. The model takes into account the direct propagation path, the first-and second-order reflected waves as well as the effect of diffraction. The utilization of linear and circular polarized antennas instead of only linear polarized antennas decreases the standard deviation of the received power and enhances the effective range. We have performed positioning with the simulated and measured powers using the mean squared error method in case of three transmitters. The average positioning error is significantly reduced by usage of the two various types of polarization antennas compared to the use of merely linearly polarized antennas.
\end{abstract}

\section{Keywords}

Indoor localization, linear and circular polarization, RSSI (Received Signal Strength Indicator)

\footnotetext{
${ }^{1}$ Department of Broadband Infocommunication and Electromagnetic Theory, Faculty of Electrical Engineering and Informatics,

Budapest University of Technology and Economics

H-1111 Budapest, P.O.B. 91, Hungary

${ }^{*}$ Corresponding author, e-mail: nagy@mht.bme.hu
}

\section{Introduction}

In our days, the determination of the position of objects and people is an important research area. Numerous location based services are based on the knowledge of the position of the subscribers. The GPS (Global Positioning System) is well usable in outdoor, however, it is not applicable in an indoor environment. For handling this problem, a lot of different methods have been developed which practically can be classified into two categories. One of these is the multilateration which is based on arrival time difference of the electromagnetic waves due to the finite propagation speed. The other one is the field strength based method which calculates the distance from the received power between the transmitter and the receiver. In this article we propose a new hardware configuration to enhance the accuracy of the latter method.

In addition to the direct propagation path, the reflected and diffracted rays are also important in indoor environment. In this case the phase-correct sum of electromagnetic wave provides the resultant field strength, which ruins the monotonicity of field strength versus distance characteristic of the free space. The received power fluctuates around a mean value varying the distance between the transmitter and receiver. The distribution of the fluctuation is Rice in case of LoS, while it is Rayleigh when there are only indirect pathways. The fluctuation of the signal level has to be reduced to enhance the accuracy of the distance measurement and as a result the efficiency of the positioning. A possible solution is the usage of directed antennas, since in this case the signals from the indirect paths are suppressed, supposing that the main directions of the transmitter and receiver antennas are faced each other [1-3]. Another option is the application of circular-polarized (CP) antennas instead of linear-polarized (LP) ones [4, 5]. After a reflection, the circularly polarized electromagnetic wave becomes circularly polarized in the opposite direction. This means that the reflected wave is cross polarized in terms of the receiving antenna, therefore, the antenna is insensitive to it. In other words, the antenna suppresses every ray suffered odd number of reflection and, thereby, the fluctuation of the received signal level is reduced. Another favourable feature of the circularly 
polarized antennas is their insensitivity to rotation in the plane perpendicular to the direction of propagation [6]. Algorithmic solution has been also developed to decrease the dependence of received signal level from the orientation of the mobile device [7]. In mobile applications it is preferred if the mobile device is equipped with omnidirectional antenna. However, the creation of the omnidirectional and circularly polarized antenna is a hard task in practice [4]. Therefore, we propose the application of circularly polarized antennas for fixed installation transmitter or receiver, while application of linearly polarized omnidirectional antennas is suggested in case of the mobile device. This solution reduces the fluctuation of the signal level arisen from the orientation of the mobile unit as well as decreases the signal variation which is resulted from the existence of indirect pathways. The proposed antenna configuration provides a $3 \mathrm{~dB}$ lower signal level than two identical polarized antennas in case of free space, however, the accuracy of the positioning is not affected. In addition, in an indoor environment the decrease of the field strength is higher-order power moving away from the transmitter in case of two linearly polarized antennas, than utilizing circular and linear polarized ones. For this reason, increasing the distance between the transmitter and the receiver, the above described power loss reduces at first, then it turns into gain.

The description of the own developed ray tracing model implemented in MATLAB environment can be found in Section 2, while the measurement and simulation environment is presented in Subsection 2.1. The Section 3 deals with measurement and simulation results. Eventually, the evaluation of accuracy of the distance measurement (positioning) is given in 3.1.

\section{The simulation model}

In order to be able to investigate the contribution of each propagation mode to the total field, we have developed a modular, quasi two-dimensional ray tracing model. In contrast to the traditional SBR (Shooting Bouncing Ray) method we have realized a direct technique. When the number of walls is lower than the number of launched rays, it is worth to suppose a path between the transmitter and receiver and to check the intersection for each wall.

The circular polarized signals can be described by the sum of two spatially orthogonal linear polarized waves with 90 degree phase distance. For this reason, a two-dimensional model is not able to demonstrate all the advantages of reception of circularly polarized waves with linearly polarized antenna. The efficiency of the model is enhanced by taking into account the first-and second order reflection from the floor and ceiling, when there is LoS between the transmitter and receiver. In NLoS scenario, the modelling can be realized only in three-dimension. In case of direct propagation way, the free space loss and phase delay have been supplemented with the additional attenuation and phase shifting of the walls [8].

$$
L_{f}=20 \lg \left(\frac{4 \pi d}{\lambda}\right)-G_{t}-G_{r}[d B]
$$

In the formula (1), $L_{f}$ is the free space loss, ' $d$ ' is the distance between the transmitter and receiver while ' $\lambda$ ' is the free space wavelength. $G_{t}$ and $G_{r}$ denote the gain of the transmitter and receiver antenna expressed in decibel. The model takes into consideration the structure of the walls, the electromagnetic features of each layer (complex permeability and permittivity), the polarization of the incident wave as well as the angle of incidence. The transmission and reflection parameters are calculated by a recursive formula [9]. The two coefficients can be expressed by the (2) (3) in case of horizontal polarization, while by the (4) (5) in case of vertical polarization.

In case of horizontal polarization:

$$
\begin{gathered}
\Gamma_{H}=\frac{E_{r}}{E_{t}}=\frac{A_{0}}{B_{0}}, \\
T_{H}=\frac{E_{t}}{E_{i}}=\frac{1}{A_{0}} .
\end{gathered}
$$

In case of vertical polarization:

$$
\begin{gathered}
\Gamma_{V}=\frac{E_{r}}{E_{i}}=\frac{C_{0}}{D_{0}}, \\
T_{V}=\frac{E_{t}}{E_{i}}=\frac{1}{D_{0}} .
\end{gathered}
$$

The ' $\Gamma$ ' is the reflection coefficient while ' $T$ ' is the transmission coefficient in the terms (2) and (3). $E_{r}$ represents the reflected electric field strength, $E_{t}$ is the transmitted wave and $E_{i}$ denotes the incident electric field. $A_{0}, B_{0}, C_{0}$ and $D_{0}$ can be expressed by (6), (7), (8) and (9).

$$
\begin{aligned}
& A_{0}=\frac{e^{\psi j}}{2}\left[A_{j+1}\left(1+Y_{j+1}\right)+B_{j+1}\left(1-Y_{j+1}\right)\right], \\
& B_{0}=\frac{e^{-\psi j}}{2}\left[A_{j+1}\left(1-Y_{j+1}\right)+B_{j+1}\left(1+Y_{j+1}\right)\right], \\
& C_{0}=\frac{e^{\psi j}}{2}\left[A_{j+1}\left(1+Z_{j+1}\right)+B_{j+1}\left(1-Z_{j+1}\right)\right], \\
& D_{0}=\frac{e^{-\psi j}}{2}\left[A_{j+1}\left(1-Z_{j+1}\right)+B_{j+1}\left(1+Z_{j+1}\right)\right] .
\end{aligned}
$$

The index ' $j$ ' refers to $j$-th layer in the formula (6), (7), (8), (9). ' $Y$ ', ' $Z$ ' and ' $\psi$ ' can be calculated by (12), (13), (14). In case of ' $\mathrm{N}$ ' number of layers, the recursive formulas can be evaluated by using the $\mathrm{N}+1$-th layer (10), (11).

$$
A_{N+1}=C_{N+1}=1 \text {, }
$$




$$
\begin{gathered}
B_{N+1}=D_{N+1}=0, \\
Y_{j+1}=\frac{\cos \left(\theta_{j+1}\right)}{\cos \left(\theta_{j}\right)} \sqrt{\frac{\varepsilon_{j+1}\left(1-j \cdot \tan \left(\delta_{j+1}\right) \cdot \mu_{j}\right)}{\varepsilon_{j}\left(1-j \cdot \tan \left(\delta_{j}\right) \cdot \mu_{j+1}\right)}}, \\
Z_{j+1}=\frac{\cos \left(\theta_{j+1}\right)}{\cos \left(\theta_{j}\right)} \sqrt{\frac{\varepsilon_{j}\left(1-j \cdot \tan \left(\delta_{j}\right) \cdot \mu_{j+1}\right)}{\varepsilon_{j+1}\left(1-j \cdot \tan \left(\delta_{j+1}\right) \cdot \mu_{j}\right)}} .
\end{gathered}
$$

In the terms of (12), (13), $\theta_{j}$ is the angle of the reflection on the $\mathrm{j}$-th layer. $\varepsilon_{j}$ denotes the permittivity of the $\mathrm{j}$-th substance, while $\tan \left(\delta_{j}\right)$ signs the loss of $\mathrm{j}$-th material. $\mu_{j}$ is the permeability of the $j$-th layer and last but not least ' $j$ ' represents the imaginary unit $(\sqrt{-1})$.

$$
\psi_{j}=d_{j} \gamma_{j} \cos \left(\theta_{j}\right)
$$

In Eq. (14), $d_{j}$ denotes the thickness of the $\mathrm{j}$-th layer and $\gamma_{j}$ can be expressed by the formula (15).

$$
\gamma_{j}= \pm \sqrt{j \omega \mu_{j}\left(\sigma_{j}+j \omega \varepsilon_{j}\right)}
$$

The ' $\omega$ ' is the angular frequency of the wave, while $\sigma_{j}$ is the conductivity of the $\mathrm{j}$-th stratum. The electrical property of the walls has a special status in terms of the model's accuracy. The dielectric constants and losses of the materials have been determined by an own-designed, coaxial resonator based measuring device [10]. Our measurement results are in good agreement with literary values. The measured complex permittivities of each wall types are presented in Table 1.

Table 1 Measured real and imaginary parts of permittivities of the various wall types $(\Re(\varepsilon), \mathfrak{I}(\varepsilon))$ at $868 \mathrm{MHz}$

\begin{tabular}{lll}
\hline Material & $\mathfrak{R}(\varepsilon)$ & $\mathfrak{I}(\varepsilon)$ \\
\hline Concrete & 7.0 & 1.2 \\
Brick & 3.7 & 0.23 \\
Glass & 6.2 & 0.01 \\
Plasterboard & 2.9 & 0.13 \\
\hline
\end{tabular}

The model takes into account not only the direct propagation path, but the reflected and diffracted waves as well, provided they only pass through less than two walls. The reason of this phenomenon is that in case of numerous transmissions, the signal energy is reduced to such an extent, that it does not contribute significantly to the resulting field. We used the UTD (Universal Theory of Diffraction) model to calculate the diffraction loss of the complex edges $[11,12]$. This type of propagation mode considerably enhances the accuracy of the model in case of NLoS. When there is LoS the contribution of the diffracted wave to the total received power is negligible due to the few transported power. The model takes into account the total three-dimensional radiation pattern of the antennas, therefore, it is suitable to investigate the effect of the directional antennas.

\subsection{The test indoor environment for simulations and measurements}

The measurements were performed at the Budapest University of Technology and Economics in a clerical environment. We have created a database based on the floor plan data which contains the $\mathrm{x}$ and $\mathrm{y}$ coordinates of the endpoints of the walls as well as the electric and magnetic parameters. That part of the database which is involved in the simulations and measurements is presented in Fig. 1. The red lines represent the walls made of brick, the black ones denote the concrete walls. The blue strips depict the windows while the purple and the green lines are the wood and metal doors. The thickest strips represent $40 \mathrm{~cm}$, the medium thick ones are $12 \mathrm{~cm}$ while the thinnest ones are $5 \mathrm{~cm}$. The measuring areas are illustrated by the light blue rectangles and lines. The green dot and triangle denote the place of the circularly and linearly polarized antennas. Both radiators were mounted at $1 \mathrm{~m}$ high as well as the measurements were performed in the same plane. Both types of antenna have been constructed as a patch so that the direction characteristic of them be the same as far as possible. The two three-dimensional direction characteristics have been constructed from the vertical and horizontal plane patterns using linear interpolation. The plane patterns of the antennas were measured in an anechoic chamber with one degree resolution. Both the simulations and measurements were performed over the $868 \mathrm{MHz}$ ISM (Industrial, Scientific and Medical) band.

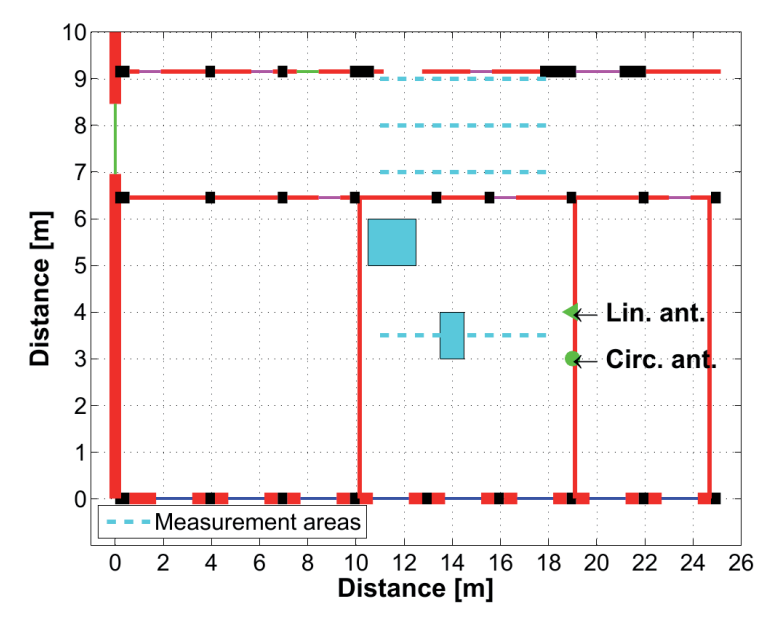

Fig. 1 Floorplan of the test environment

\section{Simulation and measurement results}

The measurements were performed with horizontal and vertical polarized receiver antenna with $10 \mathrm{~cm}$ resolution in the areas represented by light blue rectangles and dashed lines in Fig. 1. The fixed antennas transmitted $15 \mathrm{dBm}$ power sinusoidal signal. In order to be able to measure the signal of both base stations at the same time, they were tuned relative to each other by $1 \mathrm{MHz}$. 
We have performed the data acquisition with a linear-polarized omnidirectional GP (Ground Plane) antenna and spectrumanalyzer. The simulation result of the power distribution in the room which involves the light blue rectangles is shown in Fig. 2. The calculation has been executed in case of vertical polarized transmitter and receiver antennas with $10 \mathrm{~cm}$ resolution.

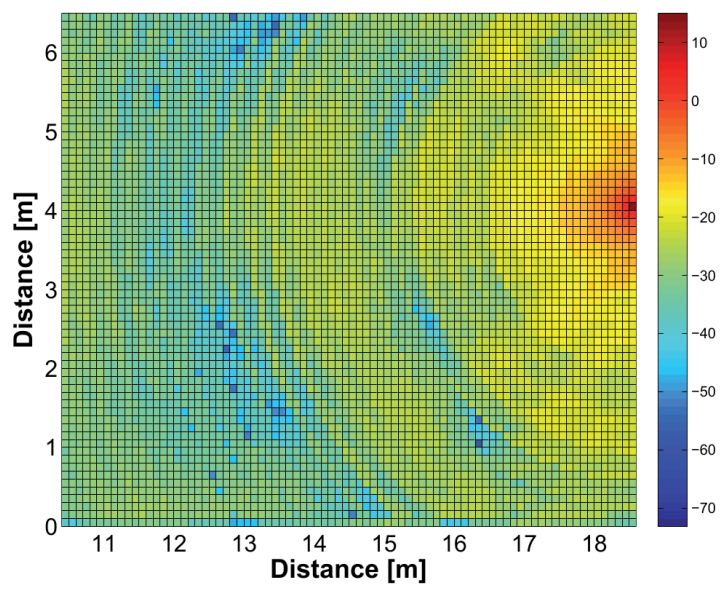

Fig. 2 The simulated receiver power, when there is LoS between the transmitter and the receiver

The engraving of the Fig. 2 prepared along the dashed blue line denoted in Fig. 1 and the measured values along the same line can be seen in Fig. 3. The features and the average slope of the two curves are in good agreement with each other. Furthermore, the Fig. 3 contains the free space loss presented by continuous red curve.

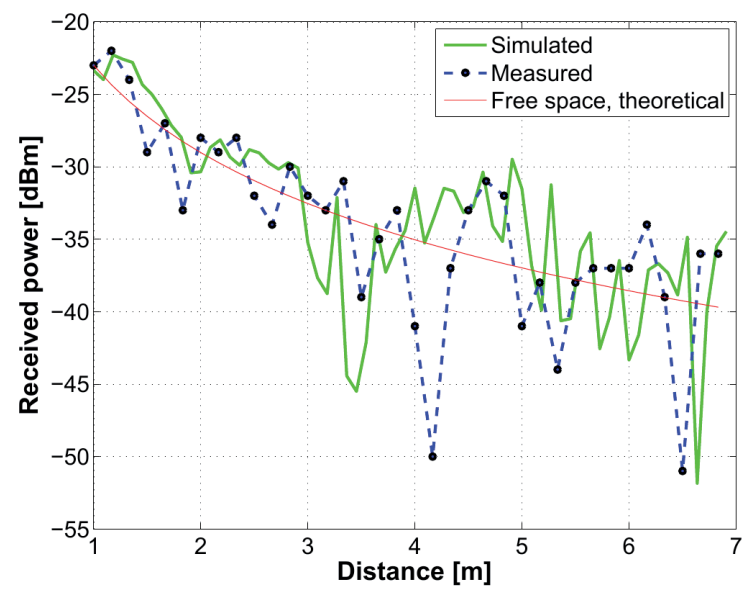

Fig. 3 Simulated and measured powers in terms of distance

The Figure 4 presents the measurement results in various polarizations which are also prepared along the dashed light blue line (Fig. 1). LP denotes the linearly (in the vertical plane), while CP signs the circularly polarized transmitter in the figure. VP and HP represent the vertically and horizontally placed (in the perpendicular plane to the direct propagation path) omnidirectional receiving antennas. As shown in the figure, the average received signal level is higher in case of circularly polarized antenna, than in case of linearly polarized transmitter in both orientation of the receiving antenna. In addition, the signal level is independent of the orientation of the receiver when the base station is circularly polarized.

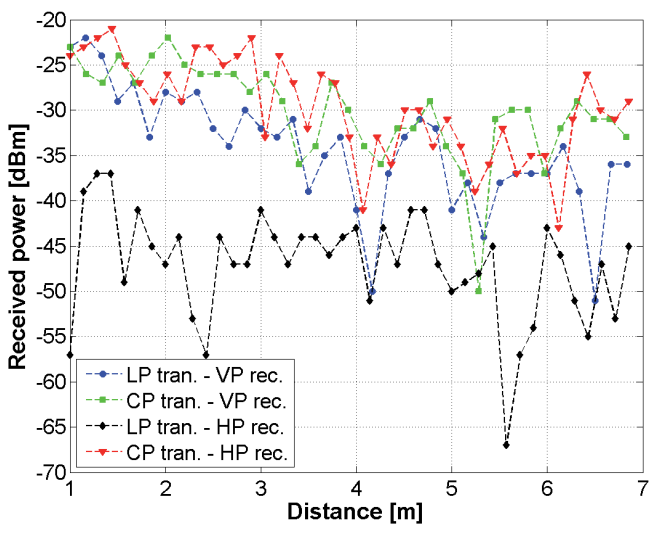

Fig. 4 The measurement of the received power, when there is LoS

Table 2 involves the standard deviation of the measured and simulated received power in LoS and NLoS scenarios.

Table 2 The standard deviation of the received power in various configuartons

\begin{tabular}{lcccccccc}
\hline & \multicolumn{3}{c}{ Simulated } & \multicolumn{5}{c}{ Measured } \\
\cline { 2 - 8 } & V-V & C-V & V-H & V-H & V-V & C-V & V-H & C-H \\
\hline LoS corner & 5.5 & 5.3 & 9.8 & 6.8 & 5.2 & 4.8 & 11.4 & 6.1 \\
LoS line & 4.1 & 3.7 & 9.4 & 6.8 & 4.4 & 3.6 & 6.7 & 5.5 \\
LoS centre & 7.6 & 6.4 & - & - & 7.4 & 6.7 & - & - \\
NLoS & 7.0 & 6.4 & - & - & 6.9 & 6.1 & - & - \\
\hline
\end{tabular}

The corner, line and centre words in Table 2 refer to the place of light blue region in Fig. 1. The notations in the second row of table correspond to the denotations of Fig. 4. The standard deviation values in Table 2 are in $\mathrm{dB}$ dimensions. We matched a logarithmic curve to the simulated and measured values utilizing LSEM (the Least Square Error Method) then we evaluated the standard deviation of difference of the matched curve and measured values. In case of rectangular region, the standard deviations of the simulated and measured values have been calculated directly. The results show that the linear polarized antenna is able to receive the circularly polarized signal with less fluctuation than the linearly polarized one in indoor environment. In addition, the average received power is also higher in term of distance.

\subsection{The accuracy of the distance measurement}

The reduction of the standard deviation using different polarized antennas means that the accuracy of the distance measurement is enhanced (Table 2). As the distance between the transmitter and receiver is estimated from more than one sample in practice, therefore we also evaluated the average of ten samples. The moving average of the sample acquired along the lines was 
calculated by a bell-curve type of weighting function. The average error of the distance measurement was $2.4 \mathrm{~m}$ using the two linear polarized antennas in LoS scenario, while the error reduced below $2.1 \mathrm{~m}$ using the circular and linear polarized antennas. The same parameters were $2.9 \mathrm{~m}$ and $2.7 \mathrm{~m}$ in NLoS scenario.

\section{Conclusion}

Based on the measurement and simulation results it can be said, that it is worth using circularly polarized antennas on the fixed base station and linearly polarized omnidirectional antennas in case of the mobile devices in indoor environment. The advantage of the solution is that the fluctuation of received power by the mobile device is reduced in compared to use only linearly polarized antennas. In addition, the received power is less sensitive to changes in orientation of the mobile antenna. A further advantage of the proposed solution is that the mobile antenna can remain omnidirectional, therefore, the sensitivity is equivalent in all directions. The summarized advantages enhance the accuracy of the RSSI based position techniques as well as the range and quality of the radio communication links.

\section{References}

[1] Szumny, R., Kurek, K., Modelski, J. "Attenuation of multipath components using directional antennas and circular polarization for indoor wireless positioning systems." In: 2007 European Microwave Conference. pp. 1680-1683. 9-12 Oct. 2007. DOI: 10.1109/eumc.2007.4405536

[2] Sato, K., Manabe, T., Ihara, T., Saito, H., Ito, S., Tanaka, T., Sugai, K., Ohmi, N., Murakami, Y., Shibayama, M., Konishi, Y., Kimura, T. "Measurements of reflection and transmission characteristics of interior structures of office building in the 60-GHz band." IEEE Transactions on Antennas and Propagation. 45 (12). pp. 1783-1792. 1997. DOI: $10.1109 / 8.650196$

[3] Fryziel, M., Loyez, C., Clavier, L., Rolland, N., Rolland, P. A. "Pathloss modell of the $60-\mathrm{GHz}$ indoor radio channel." Microwave and Optical Technology Letters. 34 (3). pp. 158-162. 2002. DOI: 10.1002/mop.10402

[4] Cidronali, A., Maddio, S., Giorgetti, G., Manes, G. "Analysis and performance of a smart antenna for $2.45-\mathrm{GHz}$ single-anchor indoor positioning." IEEE Transactions on Antennas and Propagation. 58 (1). pp. 21-31. 2010. DOI: 10.1109/tmtt.2009.2035947
[5] Huang, X. "Antenna polarization as complementarities on RSSI based location identification." In: 4th International Symposium on Wireless Pervasive Computing, 2009. ISWPC 2009. pp. 1-5, 11-13 Feb. 2009. DOI: 10.1109 /iswpc. 2009.4800570

[6] Yildirim, F., Sadri, A. S., Liu, H. "Polarization effects for indoor wireless communications at $60 \mathrm{GHz}$." IEEE Communictions Letters. 12 (9). pp. 660-662. 2008. DOI: 10.1109/lcomm.2008.080757

[7] Eltaher, A., Ghalayini, I. I., Kaiser, T. "Towards UWB Self-Positioning Systems for Indoor Environments Based on Electric Field Polarization, Signal Strength and Multiple Antennas." In: 2nd International Symposium on Wireless Communication Systems, 2005. pp. 389-393, 7-7 Sept. 2005. DOI: 10.1109/iswcs.2005.1547727

[8] de Adana, F. S., Gutierrez Blanco, O., Diego, I. G., Perez Arriaga, J., Catedra, M. F. "Propagation model based on ray tracing for the design of personal communication systems in indoor environments." IEEE Transactions on Vehicular Technology. 49 (6). pp. 2105-2112. 2000. DOI: $10.1109 / 25.901882$

[9] Balanis, C. A. "Advanced engineering electromagnetics." John Wiley \& Sons, Inc. 2012.

[10] Szalay, Z., Ther, I., Nagy, L. "Complex dielectric parameter measurement by coaxial resonator and ISM band radio module." In: Radioelektronika (RADIOELEKTRONIKA), 2013 23rd International Conference, pp. 213-218, 16-17 April 2013. DOI: 10.1109/RadioElek.2013.6530919

[11] Bernardi, P., Cicchetti, R., Testa, O. "A three-dimensional UTD heuristic diffraction coefficient for complex penetrable wedges." IEEE Transactions on Antennas and Propagation. 50 (2). pp. 217-224. 2002. DOI: $10.1109 / 8.997998$

[12] Rouviere, J.-F., Douchin, N., Combes, P. F. "Diffraction by lossy dielectric wedges using both heuristic UTD formulations and FDTD." IEEE Transactions on Antennas and Propagation. 47 (11). pp. 1702-1708. 1999. DOI: $10.1109 / 8.814950$ 